\title{
Landsliding generated by thermomechanical interactions between rock columns and wedging blocks: Study case from the Larzac Plateau (Southern France)
}

\author{
Alfredo Taboada ${ }^{1 *}$, Hadrien Ginouvez ${ }^{1^{* *}}$, Mathieu Renouf $^{2}$, Pierre Azemard ${ }^{3}$ \\ ${ }^{1}$ Laboratoire Géosciences Montpellier, UMR 5243, Université de Montpellier, France \\ ${ }^{2}$ Laboratoire de Mécanique et Génie Civil, UMR5508, Université de Montpellier, France \\ ${ }^{3}$ CEREMA, Direction Territoriale Méditerranée, Laboratoire d'Aix-en-Provence, France
}

\begin{abstract}
The Larzac Plateau is delimited by vertical cliffs whose geometry is controlled by vertical joints. Cliff's erosion involves landslides initiated by incremental enlargement of joints that progressively detach rock columns at very low velocities $(1.2 \mathrm{~mm} / \mathrm{yr})$. We find that enlargement of joints is linked to intraseasonal thermal cycles ranging between 2-15 days in relation with dilation/contraction of rock blocks trapped inside the joints. The mechanism involves two successive stages in which blocks create a wedging and a ratcheting effect on the rock column. Wedging is associated with compressional forces acting on the rock column, resulting from temperature increase and dilation of the shallow rocks. Ratcheting is associated with downward displacement of blocks by gravity to a new equilibrium position, resulting from temperature decrease and contraction of shallow rocks. The displacement vector in a thermal cycle is split into a plastic and a thermal component; plastic displacements range between $10-200 \mu \mathrm{m}$ according to the seasons, and are absorbed along a shear plane dipping $\sim 40^{\circ}$ beneath the rock column: they are largest during autumn and winter, minor during spring and negligible in summer. This deformation mechanism is termed thermomechanical creep as permanent deformations are associated to mechanical forces induced by short-term thermal cycles.
\end{abstract}

\section{Introduction}

The role of temperature fluctuations on the initiation of landslides is a controversial subject. Although thermal effects are normally considered to be secondary, recent studies suggest that temperature variations may induce permanent plastic deformations within jointed rock slopes, which may trigger landslide processes [1-3]. In particular, some authors suggest that temperature variations during annual cycles may induce shearing beneath rock columns through a mechanism combining wedging and ratcheting [4-6]. The wedging-ratcheting mechanism (which will be termed thermomechanical creep) is associated to cycles of dilation and contraction of blocks that are trapped inside the major vertical joint located between the column and the upslope cliff.

We propose a simplified theoretical model for thermomechanical creep, based on displacement and temperature measurements on a rock column, and on many field observations in the Larzac Plateau (Southern France). In our model, short-term (and not annual) thermal cycles linked to varying climatic conditions are at the origin of incremental plastic deformations beneath the rock column. Moreover, thermal cycles differ according to the seasons, inducing different plastic deformation patterns that are analysed in detail.

The paper is organized as follows: section 2 presents the geological setting of our field observations, section 3 presents data form the instrumented rock column, section 4 explains the theoretical model based on measurements and observations, and section 5 discusses the results and perspectives of this research topic.

\section{Geological and Morphological Setting}

The Larzac Plateau is primarily composed of a thick sequence of Jurassic limestone and marl layers whose thickness ranges between $1500-2000 \mathrm{~m}$; these rocks were uplifted without folding by the combined effect of large-scale mantellic processes (asthenospheric diapir) and two compressional tectonic phases (the Alpine and Pyrenean).

The plateau is dominated by thick and massive capping layers of karstified limestones and dolostones incised by a few rivers, forming river valleys and gorges whose morphologic profile is controlled by the lithology of the exposed layers: we observe rock cliffs of decametric scale along massive competent rocks $(10-100 \mathrm{~m})$, and low to intermediate slopes within marl-limestone weaker layers.

The geometry of rock cliffs is controlled by preexisting vertical joint sets. The erosion mechanisms observed along the valleys and gorges mainly involve gravitational processes such as rock falls, rock topples and rotational landslides of varying scales. The hillslopes are covered by talus deposits and soils overlying the bedrock.

\section{Instrumented Rock Column (Southern Margin of the Larzac Plateau)}

To study the influence of temperature on the stability of rock columns we have analyzed data from a rock column located in the La Lergue river valley along the cliffs that limit the upstream karstic cirque (Pas-de-l'Escalette,

*taboada@gm.univ-montp2.fr; **hadrien.ginouvez@sfr.fr 
Figure 1) [7-8]. The rock column is composed of massive dolostones (Bathonian age) underlaid by marlcarbonate weak layers with low shear strength (InfraBathonian age), enhancing the formation of landslide processes. The unstable column has a parallelepipedal shape with an approximate volume of $40000 \mathrm{~m}^{3}$ (the height, width and length are $50 \mathrm{~m}, 10 \mathrm{~m}$, and $80 \mathrm{~m}$, respectively). The column is detached from the massif by a vertical open joint striking $\mathrm{N} 130^{\circ} \mathrm{E}$. The opening of the joint ranges from $1.5 \mathrm{~m}$ at the foot of the column to $2 \mathrm{~m}$ at the top. The joint has a winding geometry with rock blocks that are blocked at different heights (Figure 1). The marl-limestone layers at the foot of the column are overlaid by a plurimetric deposit of colluvium.

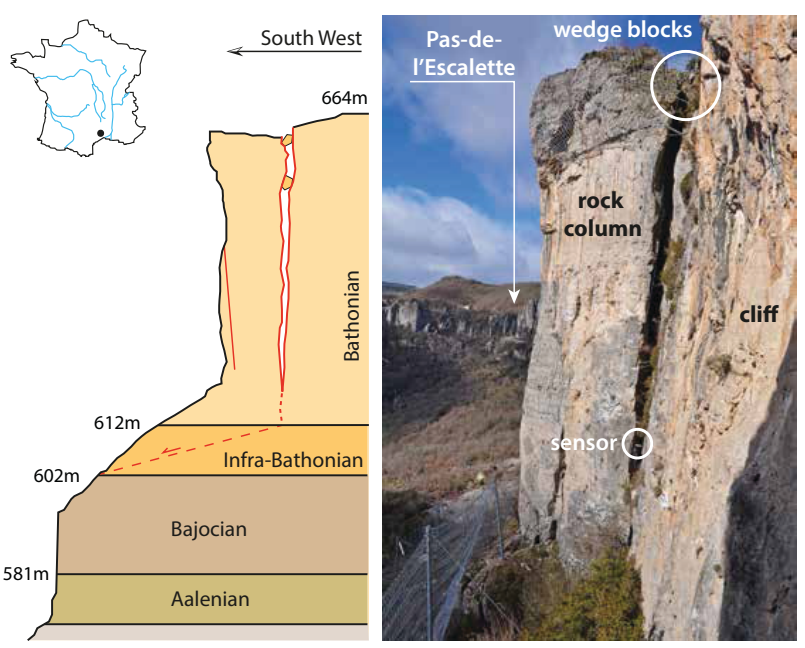

Fig. 1. Instrumented rock column showing trapped blocks within the vertical joint (Pas-de-l'Escalette, La Lergue valley).

\subsection{Kinematics of the Rock Column and Intraseasonal Temperature Cycles}

The horizontal and vertical displacements of the rock column relative to the uphill cliff were measured during a 30 months period, using high-precision displacement sensors $(0.01 \mathrm{~mm})$. The ambient temperature near the sensors was also measured to analyse possible correlations with displacements. The rock column is composed of at least 3 parts delimited by fractures, with different kinematics. The measurements from a couple of sensors located at the bottom of the open joint indicates that the central (NW) part of the column exhibits non-reversible intraseasonal displacements relative to the uphill cliff (Figure 2). These displacements are mainly observed during the autumn and winter, and to a lesser degree during the spring: cumulated horizontal and vertical displacements during an annual cycle indicate that the column moves at a rate of $\approx 1,2 \mathrm{~mm}$ toward the free boundary and dips downward. The displacements during the summer are negligible.

The trajectory of the rock column relative to the uphill cliff indicates that there is a correlation between intraseasonal temperature oscillations and displacement cycles (Figure 3). Typical non-reversible displacement cycles indicate that the joint opens as the average temperature decreases, while it tends to close as the average temperature increases (colors represent moving averages of temperature for a 48 hours interval). Note that the displacement cycle is non-reversible: the opening phase is subhorizontal while the closing phase follows a linear/concave trajectory. The average net displacement of the column during a displacement cycle is oriented toward the free boundary with a dip angle that varies according to the seasons: during the winter the displacement vector dips between $30-50^{\circ}$, whereas during the autumn it dips between $0-10^{\circ}$. The duration of thermal cycles is between 2-15 days, while average temperature variations are roughly between $1-5^{\circ} \mathrm{C}$. Thus, thermal cycles are consistent with climatic cycles observed in Southern France (e.g., 'Cévenol' storm episodes during the autumn).

\section{Simplified Theoretical Model}

We present a simplified model explaining the correlations between short-term intraseasonal displacement and thermal cycles, based on: a) the analysis of data from the instrumented column presented in the previous section ; and b) geological and morphological observations of many rock columns in the Larzac Plateau.

The model supposes that incremental enlargement of major joints is linked to intraseasonal temperature fluctuations in relation with movement of rock blocks that are blocked inside the joints. The mechanism involves two successive stages in which rock blocks create a wedging and a ratcheting effect on the rock column.

Wedging is associated with compressional forces applied on the rock column, resulting from temperature increase and dilation of shallow rock material in the uphill main cliff, the blocks and the emerged part of the rock column. On the contrary, the base of the rock column, which is often buried beneath a thick layer of talus deposits, remains at relatively constant temperature and exhibits negligible volume changes. Thermal dilation tends to close the joint inducing compressional forces in the blocks and an active lateral pressure on the rock column; if compressional forces are large enough the rock column moves outward an incremental (submillimetric) distance along a basal shear surface (e.g. a landslide) while the vertical joint is enlarged.

Ratcheting is associated with the downward displacement of blocks by gravity to a new equilibrium position, resulting from temperature decrease and contraction of the cliff - blocks - rock column system. Downward block movement sets the initial condition for the next wedging cycle as the temperature rises.

Three distinct types of thermal cycles with periods ranging between 2-15 days can be identified according to the seasons, each of which is characterized by well identified deformation cycles (Figure 4). These shortterm cycles occur during three long-term thermal phases that are easily identified in the annual temperature curve (we suppose that the long-term temperature during the year has a first-order sinusoidal shape): a) An automn 


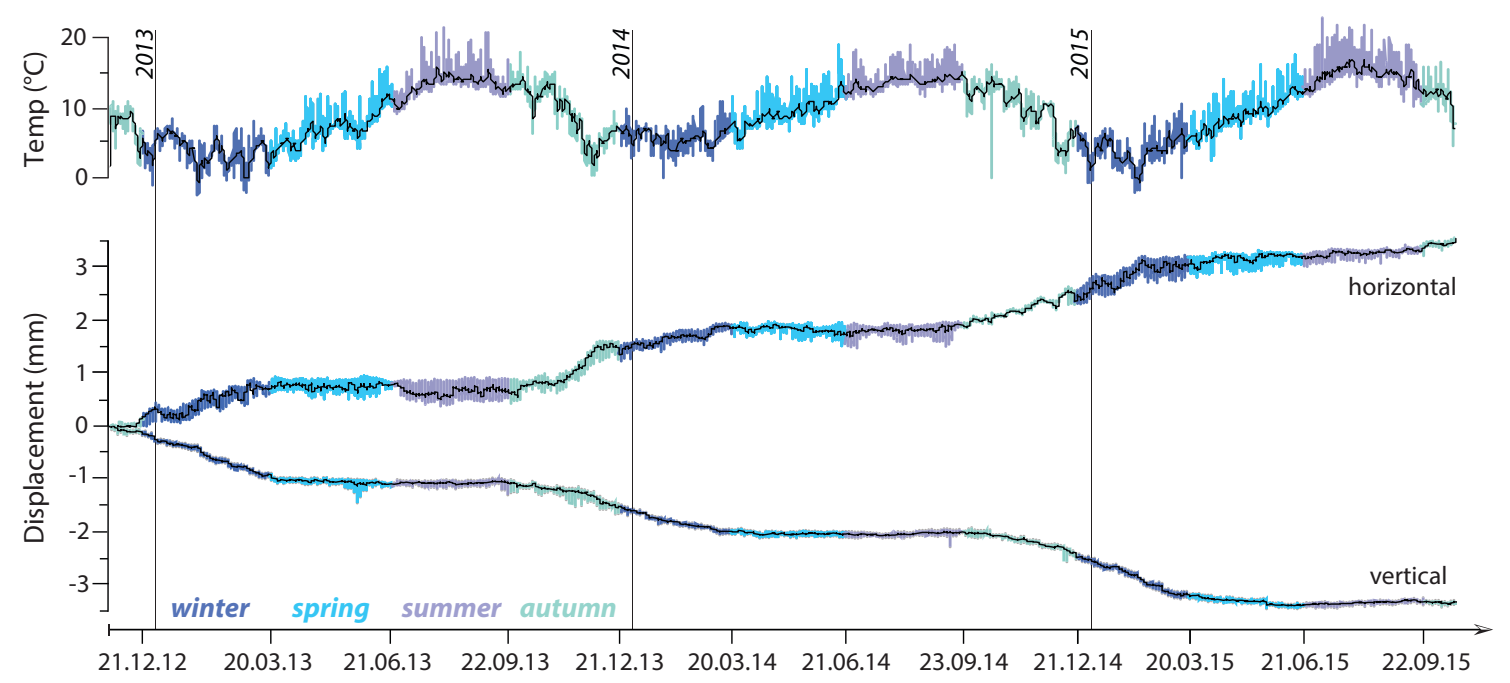

Fig. 2. Temperature and displacement data from the rock column (colored and black curves indicate continuous and moving averages over 48h) (positive horizontal displacement is toward the free boundary).

cooling phase extending from the end of summer to the end of autumn; b) A winter thermal plateau extending from the end of autumn to the end of winter; and c) A spring heating phase extending from the end of winter to the end of spring.

Note that wedging and ratcheting result from thermal fluctuations around the long-term temperatures observed during the year.

For each short-term thermal cycle the displacement vector $\delta$ is decomposed into two components, namely, a) a reversible thermal displacement $\delta T$ linked to the net variation in temperature $(T c-T a)$, without considering the wedging effect; and b) a permanent displacement $\delta p$ linked to plastic deformation at constant temperature

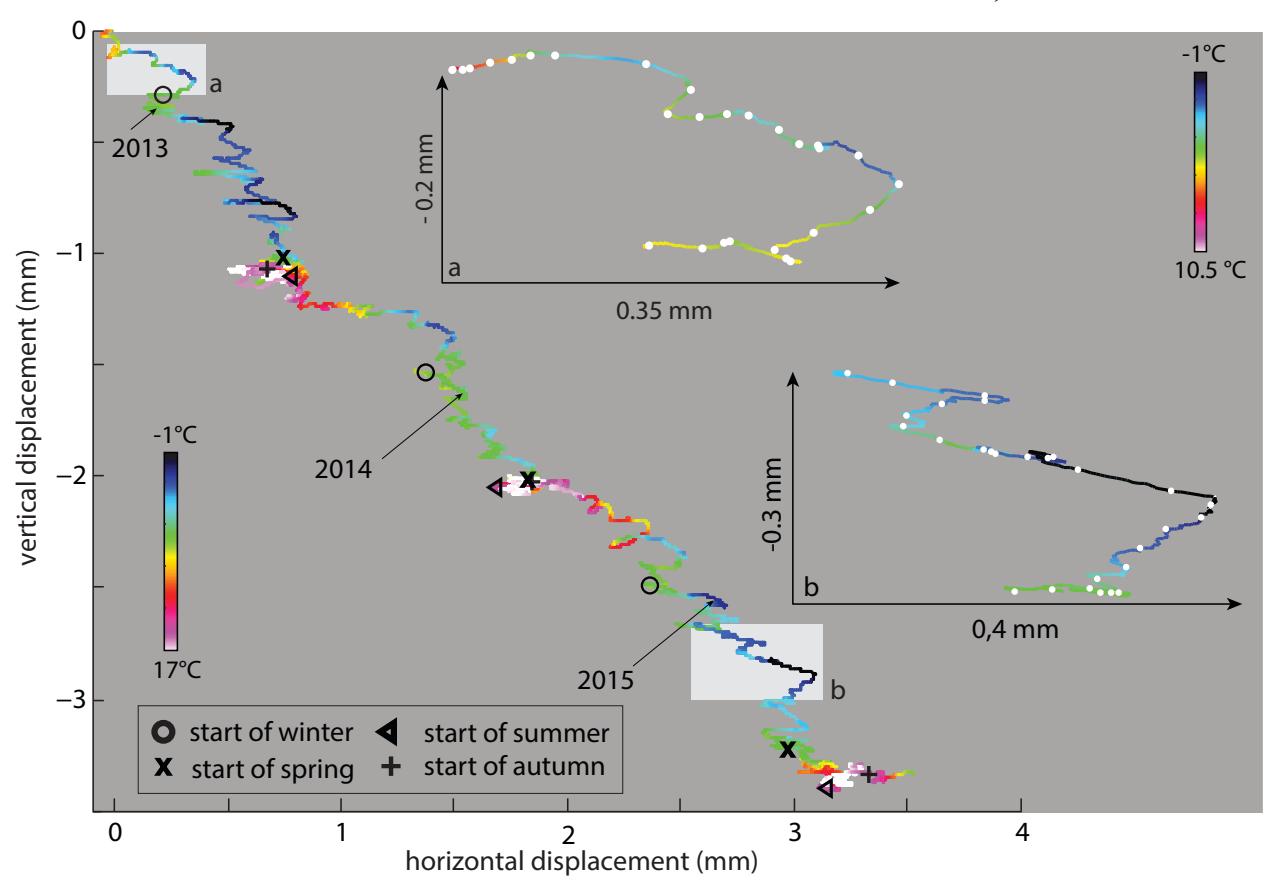

Fig. 3. Trajectory of rock column during a 30 months period (moving averages over $48 \mathrm{~h}$ ); colours indicate temperatures, (lower-left scale) (the fracture opens rightward). The two zooms (a, b) indicate intraseasonal displacement cycles (temperature $=$ upper-right scale, white circles $=$ daily intervals).
(Figure 4): Autumn thermal cycles (cooling phase) : During the autumn the long-term average temperature decreases and shallow rocks cool down progressively. Short-term thermal cycles display a high amplitude thermal decrease followed by a low amplitude thermal increase. The thermal displacement $\delta T$ is oriented toward the right (i.e., the fracture opens as temperature decreases); $\delta T$ is defined between points $a$ and $a^{\prime}$ along the trajectory, such that $T a^{\prime}=T c$. The plastic displacement $\delta p$ joins points $a^{\prime}$ and $c$ located along the trajectory and dips downward toward the free boundary.

Winter thermal cycles (thermal plateau) : During the winter the average long-term temperature remains constant, whereas short-term cooling and heating fluctuations of high amplitude are observed. Thus, the thermal displacement $\delta \quad T \quad$ is negligible and the total displacement is equivalent to the plastic displacement $(\delta=\delta p)$, which joins points $a$ and $c$ and dips at a moderate to steep angle toward the free boundary.

Spring thermal cycles (heating phase) : During the spring the long-term average temperature increases and shallow rocks heat up progressively. Short-term thermal cycles display a low amplitude thermal decrease followed by a high amplitude thermal increase. The thermal displacement $\delta \quad T \quad$ is oriented toward the left 
(i.e., the fracture closes as temperature increases). The plastic displacement $\delta p$ dips downward toward the free boundary, yet its magnitude is much smaller than in the winter cycle. This results from the reduction of the ratcheting effect, which attenuates the wedging effect.

The average displacement vector over an annual period roughly dips $40^{\circ}$ toward the free boundary and is equivalent to the plastic displacement (i.e., the cumulative thermal displacement during an annual period is negligible). This orientation can be interpreted as resulting from shearing along a basal plane located beneath the rock column.
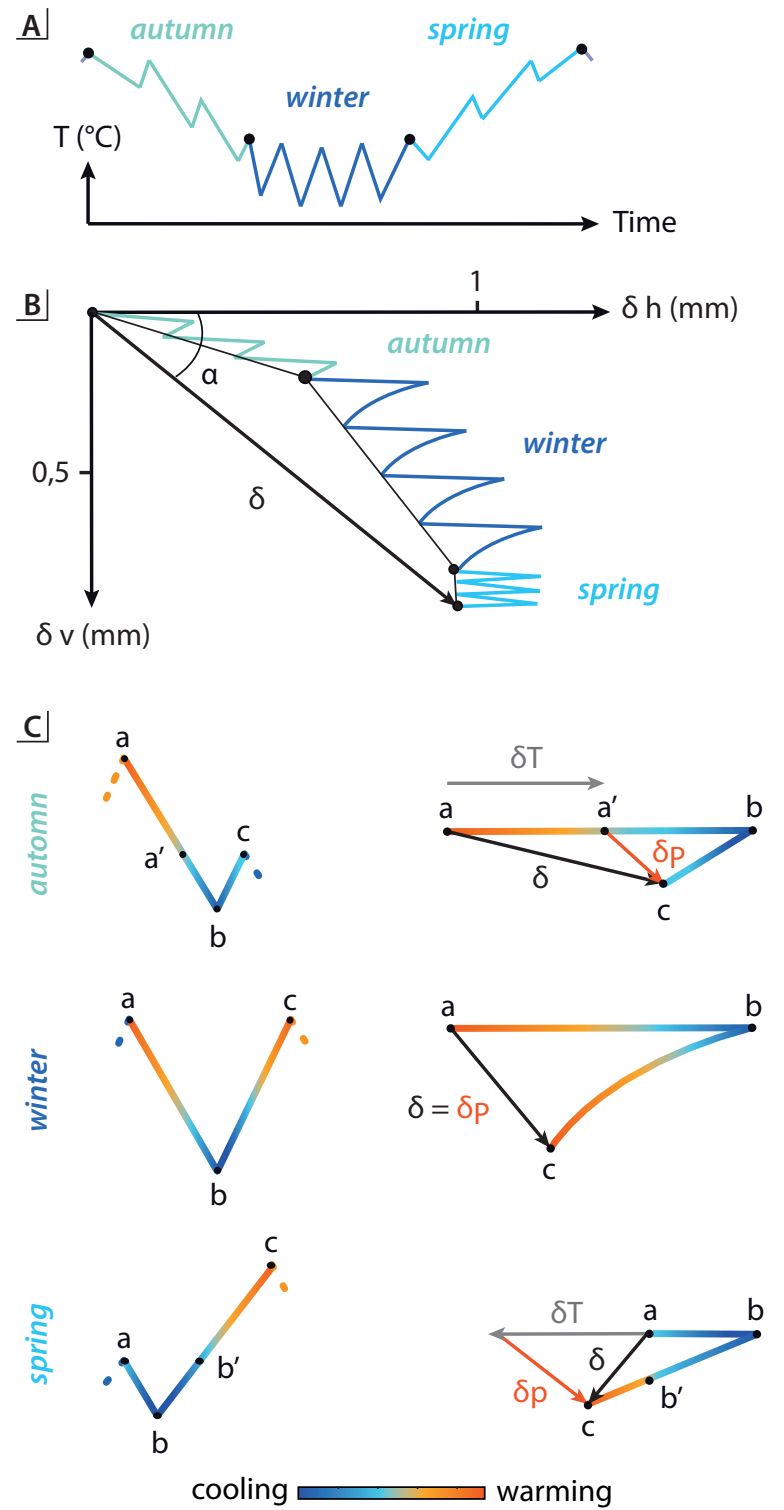

Fig. 4. Schemes illustrating temperature fluctuations and the trajectory of the rock column during an annual cycle (A, B). Displacement of the column toward the free boundary is rightward. Short-term thermal and displacement cycles of the rock column according to seasons (C).

\section{Discussion}

The analysis of displacement and temperature measurements shows that intraseasonal short-term deformation cycles of wedging and ratcheting induce incremental downslope movements of rock columns. These deformation cycles as a whole will be termed thermomechanical creep as permanent deformations are associated to mechanical forces induced by shortterm thermal cycles. As for soil creep, the finite deformation results from the sum of incremental nonreversible deformation cycles in which materials expand and contract. The balance between applied shear forces (including the gravity component) and frictional strength of rocks beneath the column controls the rate of thermomechanical creep.

We are currently performing thermomechanical simulations in order to study the influence of parameters such as the duration of short-term climatic cycles, the size of the columns relative to the blocks, and the geometry of the upslope joint, on the rate of thermomechanical creep. Many questions remain open such as the coupling of successive deformation cycles over the seasons.

\section{Acknowledgements}

This research project was financed by the programme Tellus-ALEAS, INSU CNRS (2017).

\section{References}

1. Y. Gunzburger, V. Merrien-Soukatchoffa, Y. Guglielmi, Int. Journal of Rock Mech. Mining Sc. 42, 331-349, (2005).

2. E. do A. Vargas Jr, R.Q. Velloso, L.E. Erik Chavez, L. Gusmao, C. Palmeiro do Amaral, Rock Mech Rock Eng 46 : 123-134, (2013).

3. B. @Collins, G. Stock, Nature Geoscience, 9, 395400, (2016).

4. D. Bakun-Mazor, Y.H. Hatzor, S.D. Glaser, C. Santamarina, Int. J. Rock Mech. Mining Sci. 61, 196-211, (2013).

5. C .Pasten, M. Garcia, D.D. Cortes, Géotech. Lett., 5, 186-190 (2015).

6. H. Ginouvez, Géomorphologie et dynamique de chandelles rocheuses au Pas-de-l'Escalette, geoscienstist master report, Université Montpellier, 30pp, (2015).

7. P. Azemard et al., 2009. A75 Tunnel de l'Escalette: Inspection des colonnes rocheuses dominant la tête Sud. CETE méditerranée, laboratoire régional d'Aix-en-Provence, service Géotechnique et Mécanique des Sols.

8. N. Berthoz, R. Batteux, L. Valette., 2014. A75 Lodève - Chandelles de l'Escalette : Surveillance 2014. CETE méditerranée, laboratoire régional d'Aix-en-Provence, service Géotechnique et Mécanique des Sols. 